Meta

Journal des traducteurs

Translators' Journal

\title{
Les centres de documentation spécialisés
}

\section{Lisa Galante et Lisette Ménard-Lépine}

Volume 25, numéro 1, mars 1980

La documentation

URI : https://id.erudit.org/iderudit/002785ar

DOI : https://doi.org/10.7202/002785ar

Aller au sommaire du numéro

Éditeur(s)

Les Presses de l'Université de Montréal

ISSN

0026-0452 (imprimé)

1492-1421 (numérique)

Découvrir la revue

Citer cet article

Galante, L. \& Ménard-Lépine, L. (1980). Les centres de documentation spécialisés. Meta, 25(1), 171-173. https://doi.org/10.7202/002785ar d'utilisation que vous pouvez consulter en ligne.

https://apropos.erudit.org/fr/usagers/politique-dutilisation/ 


\title{
Les centres de documentation spécialisés
}

\author{
Lisa Galante \\ documentaliste \\ LISETTE MÉNARD-LÉPINE \\ terminologue \\ Société d'Énergie de la Baie James
}

A une époque comme la nôtre, il devient indispensable aux spécialistes de diverses disciplines de se mettre au fait de l'évolution constante en matière de technologie. Il existe, comme chacun le sait, des bibliothèques spécialisées dont la raison d'être consiste justement à diffuser l'information technique pour la rendre accessible au plus grand nombre.

Ces bibliothèques appartiennent à des universités, à des sociétés industrielles ou commerciales, à des associations ou à des organismes gouvernementaux et autres. En plus des collections d'ouvrages spécialisés, on introduit de plus en plus dans les bibliothèques : des collections de périodiques, de brochures, de coupures, de cartes géographiques, de documents audio-visuels, etc. Pour désigner l'ensemble organisé de ces documents de toute nature et de toute forme, l'expression centre de documentation tend à s'implanter ; elle remplacera probablement le terme de bibliothèque qui pourra continuer à désigner ce qu'il signifie vraiment : une collection de livres.

Les centres de documentation ou «bibliothèques» mis en place par des organismes ou des sociétés se spécialisent dans la conservation et la diffusion de documents relatifs aux activités particulières à ces organisations. Ils emploient un personnel qualifié : documentalistes ou bibliothécaires et techniciens en documentation. Certains centres très spécialisés retiennent même les services d'experts en d'autres domaines; ainsi, un ingénieur peut agir à titre de recherchiste ou de conseiller pour un centre de documentation.

Les organisations qui se dotent d'un centre de documentation ont pour but de répondre à un besoin général d'information et de venir en aide aux spécialistes d'un domaine donné, dans leurs travaux de recherche et de rédaction. Le personnel des centres de documentation spécialisés se consacre à des recherches documentaires exhaustives et s'applique à rendre l'information scientifique et technique accessible. On distingue principalement trois catégories d'opérations dans un centre de documentation : l'acquisition de documents; le traitement ou le classement systématique des documents (catalogage, classification et indexation de la documentation) ; la diffusion, qui peut se faire par prêt mais qui consiste surtout à fournir des renseignements sur le fonds documentaire.

On trouve des centres de documentation spécialisés dans une foule de domaines et, comme on assiste actuellement à la création, par les P.M.E., de 
nombreux centres de documentation, l'éventail des domaines exploités s'ouvre encore davantage. Pour donner un aperçu de la diversité des sujets traités, voici une liste, très sommaire, de centres spécialisés répartis à travers le monde.

\section{ETATS-UNIS}

- American Petroleum Institute (A.P.I.).

- California Institute of Technology (CALTEC).

- Metals and Ceramics Information Center.

- Underground Excavation and Rock Properties Information Center (U.E.R.P.I.C.).

- Water Information Center.

FRANCE

- Centre d'Études et de Recherches de l'Industrie du Béton Manufacturé (C.E.R.I.B.).

- Centre de Documentation et d'Information de l'Assurance (C.D.I.A.).

- Centre du Verre B.S.N.

- Institut International du Froid (I.I.F.).

- Office de Diffusion des Panneaux de Particules (O.D.I.P.).

\section{AUTRES PAYS D'EUROPE}

- Comité Européen de l'Industrie de la Robinetterie (C.E.I.R.).

- Comité National Belge de l'Éclairage (C.N.B.E.).

- Commission Électrotechnique Internationale (C.E.I.).

Il existe des répertoires qui fournissent les nom, adresse et domaine d'activité des différents centres de documentation. Ces répertoires sont généralement présentés dans un ordre systématique et contiennent souvent des renseignements utiles concernant les services offerts par les centres de documentation. A titre d'exemple, citons :

- Special Libraries Association/Répertoire des bibliothèques spécialisées de la région de Montréal. Montréal, SLA, 1977.

- Informatech France-Québec/Répertoire des centres de documentation et des bibliothèques spécialisées du Québec. Québec, Éditeur officiel du Québec, 1974, 241p.

- Anderson, Beryl, L./Special libraries in Canada. Ottawa, Canadian Library Association, 1968, 217p.

- Fyfe, Janet/Directory of special collections in Canadian libraries. Ottawa, Canadian Library Association, 1968, 2v. 
- Dictionary of special libraries and information centers. 2d ed. Detroit, Gale Research Company, 1968, 1048p.

- France. Bibliothèque nationale/Répertoire des bibliothèques d'étude et organismes de documentation. Paris, La Bibliothèque, 1963, 3 vol.

- International library directory. 3d ed., 1969/70. London, Wales, 1968, 1221p.

Il faut aussi mentionner quelques organismes clés qui, dans la plupart des cas, réussissent à repérer les documents rares dont un spécialiste peut avoir besoin.

- Bibliothèque nationale du Québec.

- Informatech France-Québec.

- Bibliothèque nationale du Canada.

- Bibliothèque scientifique nationale du Canada.

- Conseil national de recherches du Canada (C.N.R.C.).

- Institut canadien de l'information scientifique et technique (ICIST).

- Library of Congress (États-Unis).

- Association Française de Normalisation (AFNOR) (France).

- Bureau National de l'Information Scientifique et Technique (France).

- Centre National de la recherche scientifique (C.N.R.S.) (France).

- British Museum (Grande-Bretagne).

- International Standardization Organization (ISO) (Suisse).

Pour des raisons d'efficacité, les centres de documentation communiquent entre eux en respectant une marche à suivre. Toute personne désirant communiquer avec l'un de ces centres aura donc intérêt à agir par l'intermédiaire du centre de documentation de son employeur, s'il y a lieu, ou encore à s'adresser à l'Institut canadien de l'Information scientifique et technique (ICIST) ${ }^{1}$. Le personnel de cet organisme saura orienter la recherche documentaire et fera les démarches nécessaires pour obtenir les documents requis.

En fait, le travail de recherche documentaire, comme tout autre travail de recherche, doit être fait de façon méthodique et avisée. Qu'il s'agisse de cerner avec précision le domaine de la recherche, ou encore de repérer les documents appropriés, les conseils éclairés d'un spécialiste de la documentation et la consultation des centres de documentation spécialisés éviteront bien des tâtonnements.

1. Institut canadien de l'information scientifique et technique (ICIST), Conseil national de recherches du Canada, Edifice M 55, Ottawa (Ontario) K1A 0S2. 